\title{
Parry Romberg Syndrome: An Unusual Clinical Diagnosis
}

\author{
${ }^{1}$ Gaurav Ashish, ${ }^{2}$ Mary John, ${ }^{3}$ Naina Picardo, ${ }^{4}$ Mary Kurien
}

\begin{abstract}
Parry Romberg syndrome is a very rare clinical entity, manifested by chronic progressive hemifacial atrophy. The exact pathophysiology is poorly understood; however many hypotheses have been put forward to explain the above condition. It is a self-limiting condition and usually settles down after a few years of active facial resorption.

This can clinically overlap with linear scleroderma also known as "en coup de sabre," which is seen as a diagonal pit in the frontoparietal area. When the active phase is over, various treatment modalities have been employed such as fat, silicone or bone implants and pedicled flaps but with limited success. We present a case of Parry Romberg syndrome with involvement of sagittal sutures of the skull, an association that has been rarely reported. This case is being reported with aim of better understanding of this clinical entity regarding diagnosis and management.
\end{abstract}

Keywords: Blaschko's lines, En coup de sabre, Hemifacial atrophy, Parry Romberg syndrome.

How to cite this article: Ashish G, John M, Picardo N, Kurien M. Parry Romberg Syndrome: An Unusual Clinical Diagnosis. Int J Otorhinolaryngol Clin 2017;9(3):109-111.

Source of support: Nil

Conflict of interest: None

\section{INTRODUCTION}

Parry Romberg is a clinical entity introduced by Caleb Parry in 1815, and later popularised as a syndrome by Romberg in $1846 .{ }^{1}$ Progressive atrophy of the skin and subcutaneous tissue from one side the face which may even involve the bony or cartilaginous components are the hallmark features of this clinical condition. This is a progressive but self-limiting condition. ${ }^{2}$

It is usually seen in the first or second decade of life. The clinical manifestations are more severe if presents at a younger age group. Apart from the esthetic manifestations of psychological, ocular, migraine, neuralgia and

${ }^{1}$ Postgraduate Registrar, ${ }^{2}$ Associate Professor, ${ }^{3}$ Assistant Professor, ${ }^{4}$ Professor

${ }^{1}$ Department of ENT and Head and Neck, Christian Medical College, Vellore, Tamil Nadu, India

${ }^{2-4}$ Department of ENT, CMC Vellore, Tamil Nadu, India

Corresponding Author: Gaurav Ashish, Postgraduate Registrar, Department of ENT and Head and Neck, Christian Medical College, Vellore, Tamil Nadu, India, e-mail: eauravashish05@ gmail.com seizures are the other possible clinical entities related to its clinical constellation. ${ }^{3}$

The cause is unknown for this rare condition. Genetic predisposition, autoimmune causes, endocrine causes, history of trauma, cerebral mediated alteration in fat metabolism and viral infections are few of the proposed hypothesis. However, none of the above-proposed explanations are conclusive. ${ }^{4,5}$

Definitive treatment is to be considered once the active phase is over. Usually, this involves a multidisciplinary approach including ENT surgeon, plastic surgeon, dental surgeon, physician and psychologist. ${ }^{6}$

\section{CASE REPORT}

Thirty-one-year-old gentleman presented to our tertiary care hospital with a history of progressive hemiatrophy of face on the right side for the last 4 years. No history of seizures, migraine, trigeminal neuralgia or hemifacial spasm was present. There was no history of trauma or any other major illness in the past. Physical examination suggested marked wasting of the right side of the face with atrophy of skin and subcutaneous tissue (Fig. 1). The overlying skin was normal. The right side of the tongue was also atrophied when compared to the left side (Fig. 2). The linear depressed pit in the mid-frontal region extending up to sagittal suture was evident which has been documented as "en coup de sabre" which means, "cut with the sword" in French (Fig. 3). Dental and neurological examinations were normal. CT and MRI scan revealed atrophy of right side of the face involving malar area,

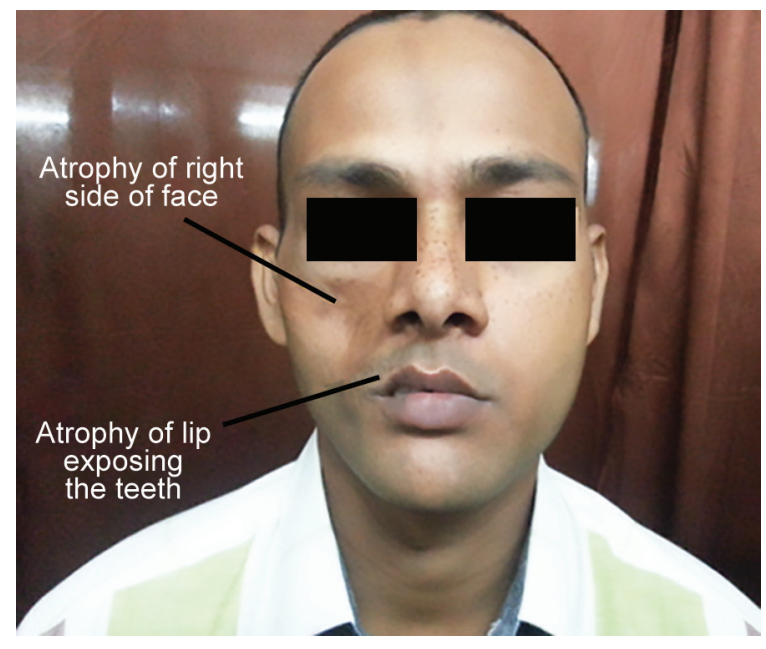

Fig. 1: Right hemifacial atrophy 


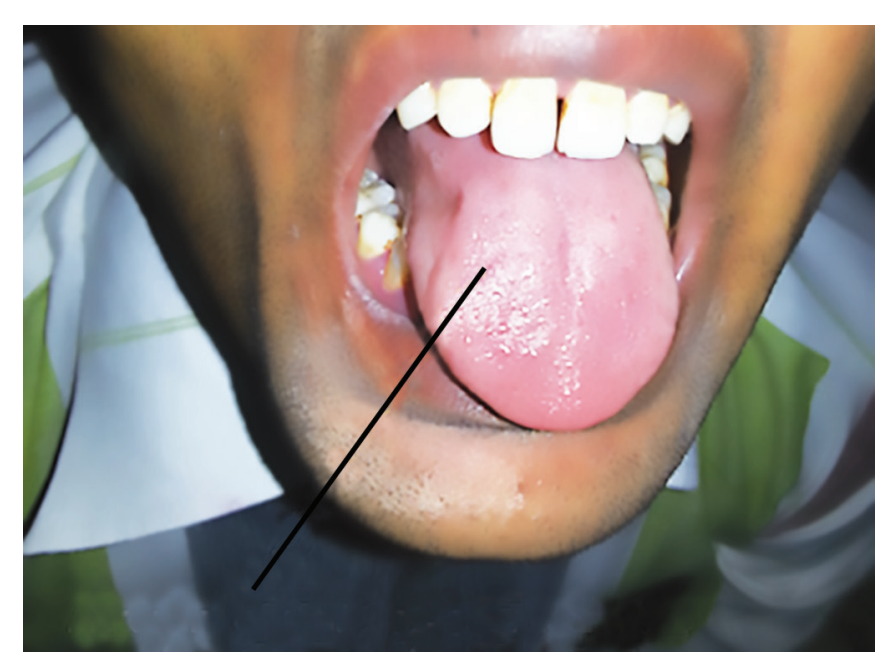

Fig. 2: Atrophy of right tongue

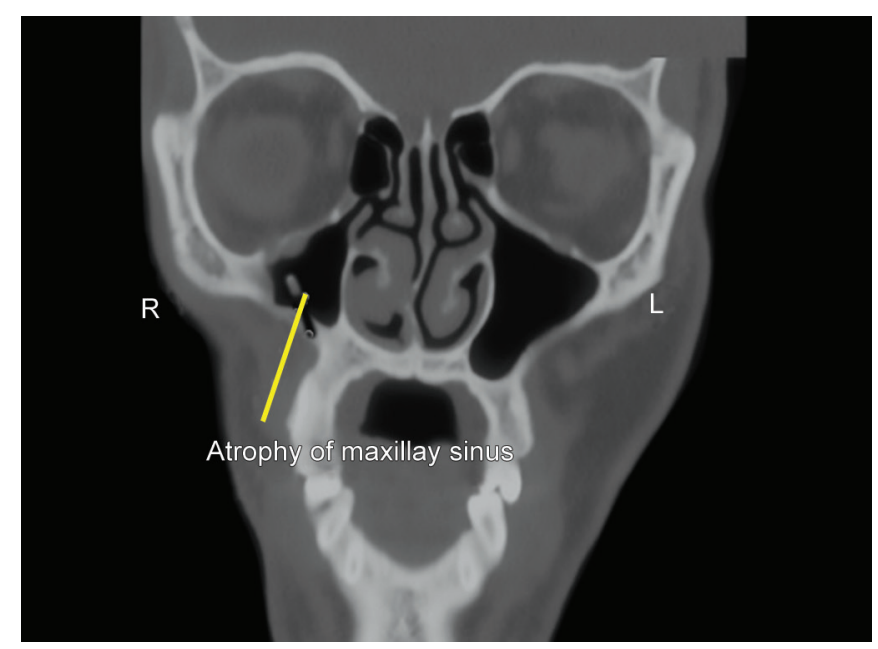

Fig. 4: CT coronal view

skin, subcutaneous tissue, masseter muscle and maxilla (Figs 4 and 5). Immunological and autoimmune workup was normal. Various treatment options were then explained to the patient. However, as the disease process was active, he opted to wait until it attained a plateau phase.

\section{DISCUSSION}

Parry Romberg syndrome (PRS) is a poorly understood clinical entity which manifests as a slow and progressive hemifacial atrophy involving the muscles, bones, subcutaneous tissue and skin. It is more common in females, usually involving the left side of the face with the highest incidence in the second decade of life. ${ }^{7}$

Due to the rarity of this syndrome, the estimated prevalence is approximately 1 per 700000 in the general population. ${ }^{8}$ Usually, this condition has an active phase where slow but consistent resorption of the hemifacial structures occurs. Later it stabilizes to a plateau phase where there is no further atrophy of facial structures. Any treatment for this condition has been suggested only after

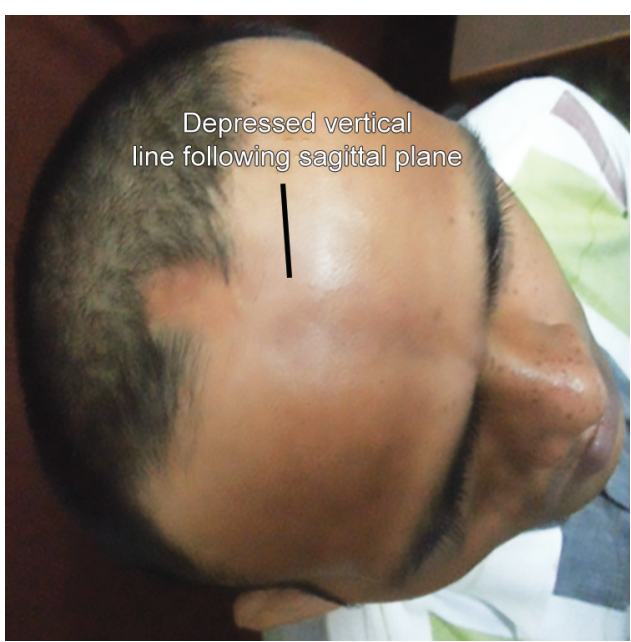

Fig. 3: En coup de sabre

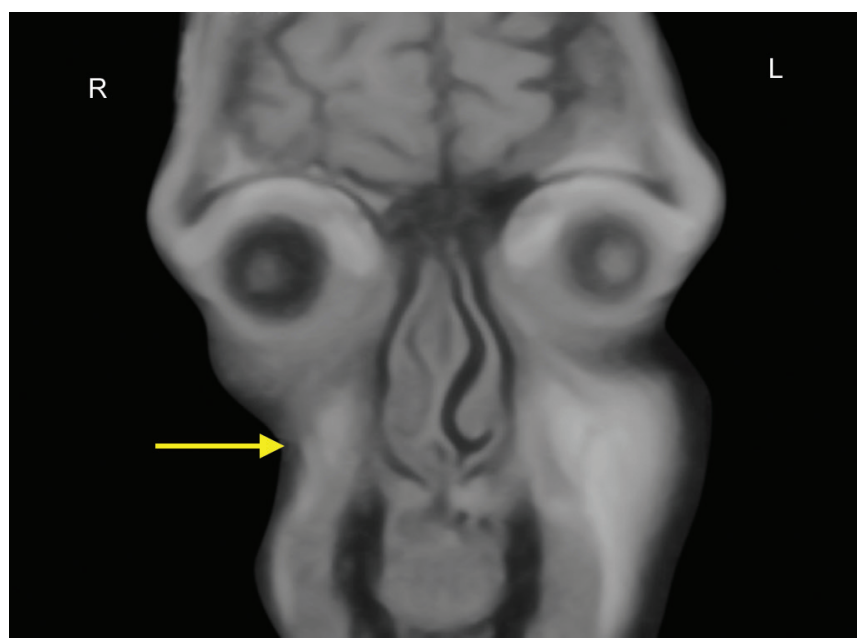

Fig. 5: Arrow showing (MRI) atrophy of skin, subcutaneous tissue and muscle

the plateau phase has been attained. ${ }^{6}$ It is usually limited to one half of the face and rarely also involves the whole half of the body on the same side. The involvement of bilateral structures has been reported as around 10-23\% of the diagnosed cases. ${ }^{9}$

These patients have a diverse clinical presentation. These manifest as hemiatrophy of the face, hemiatrophy of the tongue (approximately 25\%) and hemiatrophy of the ipsilateral or contralateral arm, trunk or leg (20\%). It can lead to deviation of face to ipsilateral side along with deviation of nose and exposure of some teeth due to involvement of lips. ${ }^{10}$ Patients can also present with features of a migraine, facial pain, trigeminal neuralgia or epilepsy. These can also have dental abnormalities such as hemimasticatory spasm and trismus. Ocular involvement such as enophthalmos, uveitis, papillary abnormalities, and ocular myopathy has also been reported. Vitiligo and other skin and hair pigmentation disorders are often associated with the above clinical entity. ${ }^{5,8}$

The junction at which the atrophied side meets the normal side may be manifested as a line. Approximately, 
$25 \%$ of patients afflicted with hemifacial atrophy have a vertical or diagonal line over the forehead which is a result of cutaneous sclerosis. These usually follow the same patterns of the "Blaschko's lines." ${ }^{\text {"11 }}$ These lines are vertically and diagonally oriented lines on the forehead, and many skin diseases follow these lines. Occasionally, a pathological line may be present on the forehead as a result of limited cutaneous sclerosis, and this is an overlapping entity with Parry Romberg syndrome. This line is called scleroderma "en coup de sabre" because this looks like a depression with the linear pit as a result of a sword injury over the forehead. ${ }^{1,5,7,10}$

Various theories have been proposed to explain the above rare clinical entity suchastrauma, encephalitis, autoimmune, infective, vasculitis, scleroderma, malformative and genetic have been put forward to explain its occurrence. However, a multifactorial affliction may be the most acceptable proposed hypothesis. ${ }^{2,3,8}$

Hemifacial microsomia (first and second branchial arch syndrome), Goldenhar syndrome, and atrophy as a result of trauma and partial lipodystrophy also known as Barraquer-Simon syndromes are few of the common differentials to be considered while diagnosing PRS.

Radiological features that may be associated are MRI brain abnormalities on the same or opposite side. MRI or CT scan show hemifacial skeletal atrophy involving maxilla and mandibular bones. Rarely maxillary sinus may be also of small size when compared to the other side. The muscles, subcutaneous tissue, and skin also show atrophy. Imaging may also reveal dental abnormalities and pathologies. ${ }^{8}$

Treatment, as mentioned previously, is to be only attempted once the disease is stable. ${ }^{6}$ The various restorative plastic surgery options include silicone implants, fat implants, pedicled flaps or bone implants. ${ }^{12}$ Medical therapy with limited success includes initiating steroid therapy, methotrexate, aziathoprine, and cyclophosphamide. Bovine collagen and inorganic implants are few other treatment options. ${ }^{13,14}$ However multiple sittings, resorption over a period and financial implications are the limiting factors for the surgical treatment. The treatment is multidisciplinary involving plastic surgeon, physician, dental surgeon, otorhinolaryngologist, ophthalmologist and often a psychiatrist.

\section{CONCLUSION}

Parry Romberg syndrome remains a diagnostic and therapeutic challenge for clinicians due to its uncommon presentation, unexplained cause and lack of definitive treatment with a satisfactory outcome. This condition can be overlapping with a clinical condition "coup de sabre" which is a form of limited sclerosis. This case is being presented with the aim of developing an insight in the diagnosis and treatment of such a rare clinical identity.

\section{REFERENCES}

1. Padmini P, Singhi PD. Facial hemiatrophy. Indian Pediatr 1992;29(4):505-507.

2. Gulati S, Jain V, Garg G. Parry Romberg syndrome. Indian J Pediatr 2006;73(5):448-449.

3. Patel H, Thakkar C, Patel K. Parry-romberg syndrome: a rare entity. J Maxillofac Oral Surg 2010;9(3):247-250.

4. Foster TD. The effects of hemifacial atrophy on dental growth. Br Dent J 1979;146(5):148-150.

5. Pinheiro TP da S, Silva CC da, Silveira CSL da, et al. Progressive hemifacial atrophy-case report. Med Oral Patol Oral Cir Bucal 2006;11(2):E112-E114.

6. Deshingkar SA, Barpande SR, Bhavthankar JD, et al. Progressive hemifacial atrophy (Parry-Romberg Syndrome). Contemp Clin Dent 2012;3(Suppl1):S78-S81.

7. Moore MH, Wong KS, Proudman TW, et al. Progressive hemifacial atrophy (Romberg's disease): skeletal involvement and treatment. Br J Plast Surg 1993;46(1):39-44.

8. Stone J. Parry-Romberg syndrome. Pract Neurol 2006; 6(3):185-188

9. Miller NR, Walsh FB, Hoyt WF. Walsh and Hoyt's Clinical Neuro-ophthalmology. Lippincott Williams and Wilkins; 2005. p. 1404.

10. Pensler JM, Murphy GF, Mulliken JB. Clinical and ultrastructural studies of Romberg's hemifacial atrophy. Plast Reconstr Surg 1990;85(5):669-674; discussion 675-676.

11. Nair PA, Patel NH, Diwan NG. Parry-Romberg Syndrome: A Linear Variant of Scleroderma with Discoid Lupus Erythematosus on Scalp-An Association. Int J Trichology 2014;6(2):71-74.

12. De la Fuente A, Jimenez A. Latissimus dorsi free flap for restoration of facial contour defects. Ann Plast Surg 1989;22(1):1-8.

13. Donofrio LM. Panfacial volume restoration with fat. Dermatol Surg Off Publ Am Soc Dermatol Surg Al 2005;31(11 Pt 2):1496-1505.

14. Roddi R, Riggio E, Gilbert PM, et al. Clinical evaluation of techniques used in the surgical treatment of progressive hemifacial atrophy. J Cranio-Maxillo-fac Surg Off Publ Eur Assoc Cranio-Maxillo-fac Surg 1994;22(1):23-32. 